\title{
TIPS TO MANIPULATE MYOGENESIS: RETENTION OF MYOBLAST DIFFERENTIATION CAPACITY USING MICROSPHERE CULTURE
}

\author{
N. Parmar and R. M. Day* \\ Applied Biomedical Engineering Group, Division of Medicine, University College London, Rockefeller Building, \\ 21 University Street, London WC1E 6JJ, UK
}

\begin{abstract}
Cell therapy is an emerging option for regenerating skeletal muscle. Improved delivery methods for anchoragedependent myoblasts are likely to improve integration and function of transplanted muscle cells. Highly porous microspheres, produced using thermally induced phase separation (TIPS), have features ideally suited for minimally invasive cell delivery. The purpose of this study was to investigate, for the first time, the use of TIPS microspheres as highly porous microcarriers for manipulation of human skeletal muscle myoblasts (HSMM) under defined culture conditions. HSMM cells readily attached to the surface of poly (DL-lactide-coglycolide) (PLGA) TIPS microcarriers, where they were induced to continue proliferating or to be driven towards differentiation whilst under static-dynamic culture conditions for 7 days. Switching from proliferation medium to differentiation medium for 7 days, resulted in increased protein expression of skeletal muscle cell contractile apparatus components, MyoD and skeletal muscle myosin heavy chain, compared with cells cultured on conventional culture plasticware for the same duration $(p<0.001)$. Growth of myoblasts on the surface of the microcarriers and their migration following simulated delivery, caused no change to the proliferative capacity of cells over 7 days. Results from this study demonstrate that TIPS microspheres provide an ideal vehicle for the expansion and delivery of myoblasts for therapeutic applications. Transplantation of myoblasts anchored to a substrate, rather than in suspension, will reduce the amount of ex vivo manipulation required during preparation of the product and allows cells to be delivered in a more natural state. This will improve the ability to control cell dosage and increase the likelihood of efficacy.
\end{abstract}

Keywords: Cell therapy, microcarrier, TIPS microspheres, skeletal muscle, biomaterials, proliferation, differentiation.

\footnotetext{
*Address for correspondence:

Richard M Day

University College London

Gower Street

London, WC1E 6BT, UK
}

Telephone: +44 (0) 2076792000

Email: r.m.day@ucl.ac.uk

\section{Introduction}

Cell therapy is an emerging treatment for regenerating tissue affected by skeletal muscle disease and volumetric muscle loss. Culture of cells from a biopsy, for autologous transplantation, is an established process for regenerative medicine based therapies, with autologous cell therapy circumventing complications associated with host immune rejection. For skeletal muscle regeneration, autologous myoblasts are isolated, cultured and expanded to provide a quantity of cells estimated to be sufficient to achieve restoration of tissue function. Minimal manipulation of cells ex vivo, to produce the final product for therapeutic application, is preferable from an industrial and regulatory viewpoint. For example, centrifugal force, cryopreservation and antibiotic treatment are routinely used in conventional cell culture but may have implications on the use of cells intended as a therapeutic product (Sekiya et al., 2012).

Myoblast engraftment and survival are fundamentally governed by the cell niche. In particular, the extracellular matrix (ECM) provides a complex structure that supports cell adhesion, anchorage and facilitates signalling to direct cell fate via dynamic cell-matrix interactions (Chiron et al., 2012). Detachment and transplantation of cultured anchorage-dependent cell types, in a suspended non-anchored state, may be detrimental to fulfilling their myogenic potential. The interaction between cell adhesion proteins, including integrins, M-cadherins and vascular cellular adhesion molecule 1 (VCAM1), and myogenic differentiation is closely regulated (Costa, 2014; Przewozniak et al., 2013). The role of integrins in myoblast maturation includes cell fusion and participation in myofibre cytoskeletal assembly during embryogenesis (Schwander et al., 2003). It is therefore likely that myoblast behaviour following transplantation will also be influenced by their anchorage state.

The physical properties of scaffold structures also influence the behaviour of myoblasts (Kilian et al., 2010). Surface topography, microstructure and mechanical properties may affect cell adhesion, proliferation and differentiation (Knight et al., 2013). The use of spherical microcarriers is a widely used technique to expand anchorage-dependent cells in culture (Yao et al., 2013; Kang et al., 2008). Microcarriers provide a large surface area for cell adhesion and proliferation in vitro. Additionally, they offer a temporary niche following in vivo delivery whilst cells are still attached to the microcarrier. This may be advantageous over cell expansion on conventional tissue culture substrates, which often necessitates chemical and/ or physical detachment from the culture vessel prior to in vivo delivery. Cell detachment disturbs adhesion to extracellular matrix proteins and may damage the cell 
membrane, resulting in reduced viability and suboptimal cellular products (Gilbert et al., 2006; Ahmadi et al., 2011).

We have developed a novel polymeric microsphere technology for use as a minimally invasive scaffold and delivery system for cells and drugs (Blaker et al., 2008a; Blaker et al., 2008b; Ahmadi et al., 2011). The microspheres are composed of poly (D, L-lactide-coglycolide) (PLGA), a biocompatible and biodegradable material, and are prepared using thermally induced phase separation (TIPS).

The aim of the current study was to investigate the attachment of primary cultures of human skeletal muscle myoblasts to TIPS microcarriers under specific culture conditions that induce either cell proliferation or differentiation, with a view to using cellularised TIPS microspheres as a therapeutic system.

\section{Materials and Methods}

\section{Manufacture of TIPS microspheres}

PLGA TIPS microcarriers were prepared as previously described (Ahmadi et al., 2011). Poly (D, L-lactideco-glycolide) 75:25 (Purasorb PDLG7507, Purac Biomaterials, Gorinchem, Netherlands) was dissolved in dimethylcarbonate $(1: 24(\mathrm{w} / \mathrm{v}))$ (Sigma-Aldrich) using magnetic stirring overnight. The polymer solution was fed into a Nisco Encapsulator Unit (Nisco Engineering, Zurich, Switzerland) by a syringe pump (Harvard Apparatus, Kent, UK), connected by a silicone tube, at a constant rate of $3 \mathrm{~mL} \mathrm{~min}$. The vibration frequency of the nozzle was kept at $1.80 \mathrm{kHz}$ and the amplitude of frequency at $100 \%$. The polymer droplets were delivered through a $150 \mu \mathrm{m}$ sapphire nozzle.

Polymer droplets were collected in a polyethylene beaker containing $250 \mathrm{~mL}$ of liquid nitrogen. The frozen droplets were equilibrated in the liquid nitrogen, transferred to $50 \mathrm{~mL}$ Falcon $^{\mathrm{TM}}$ tubes (BD Biosciences, Oxford, UK) and stored frozen at $-80{ }^{\circ} \mathrm{C}$ for $10 \mathrm{~min}$. Samples were freeze-dried (MicroModulyo, Fisher Scientific) for $24 \mathrm{~h}$ to allow sublimation of residual dimethylcarbonate. For the experiments conducted in the current study PLGA TIPS microcarriers were sieved to produce microspheres with a mean diameter of $337.5 \pm 87.5 \mu \mathrm{m}$.

Attachment of human skeletal muscle myoblasts to TIPS microspheres

Microspheres were added to $1.5 \mathrm{~mL}$ microfuge tubes (Eppendorf, Hamburg, Germany) at $80 \pm 5$ per tube. Industrial methylated spirit contains $99 \%$ ethanol and $1 \%$ methanol, which was used as an alternative to pure ethanol for this set of experiments. A solution of $70 \%$ (v/v) industrial methylated spirit (IMS) (99\% ethanol, $1 \%$ methanol) in deionised water and proliferation medium $(1: 5 \mathrm{v} / \mathrm{v})$ was added to the microspheres sequentially and centrifuged at $14,000 \mathrm{rpm}$, followed by incubation at $37^{\circ} \mathrm{C}$ for $18 \mathrm{~h}$.

Human primary human skeletal muscle myoblast cells (Lonza, Basel, Switzerland) were cultured in proliferation medium consisting of Ham's F10 medium (Gibco, Carlsbad, USA) containing $20 \%$ foetal bovine serum (FBS) (Gibco, Carlsbad, USA), $100 \mathrm{U} / \mathrm{mL}$ penicillin and $100 \mu \mathrm{g} / \mathrm{mL}$ streptomycin, $0.25 \mu \mathrm{g} / \mathrm{mL}$ amphotericin B (Sigma-Aldrich, Dorset, UK), $10 \mathrm{ng} / \mathrm{mL}$ basic fibroblast growth factor (bFGF) (Peprotech, New Jersey, USA) and incubated at $37^{\circ} \mathrm{C}, 5 \% \mathrm{CO}_{2}$.

For HSMM cell attachment to TIPS microspheres, cells were trypsinised, counted and adjusted to $1 \times 10^{6}$ cells per $1 \mathrm{~mL}$. The culture medium-alcohol solution was removed and the microspheres were washed twice with $500 \mu \mathrm{L}$ PBS before adding $500 \mu \mathrm{L}$ proliferation medium. Microspheres in medium were transferred into individual wells of a 96-well ultra-low attachment plate (Corning, New York, USA). A cell suspension of 40,000 cells in a total volume of $100 \mu \mathrm{L}$ was added to each well containing microspheres and incubated at $37^{\circ} \mathrm{C}, 5 \% \mathrm{CO}_{2}$ on a microplate shaker (SciQuip, Shropshire, UK). The plate was shaken for $1 \mathrm{~min}$ at $250 \mathrm{rpm}$. The plate was subsequently shaken at the same speed for $1 \mathrm{~min}$ per $\mathrm{h}$ throughout the incubation period.

\section{Culture of HSMM on TIPS microspheres in proliferation and differentiation medium}

PLGA TIPS microspheres and cells were prepared for attachment and incubation in proliferation medium as described above. Cells were maintained for 1 or $7 \mathrm{~d}$ in $100 \mu \mathrm{L}$ of proliferation medium under static-dynamic incubation conditions with the culture medium being replaced every $2 \mathrm{~d}$ (Pavlath, 1996) (Table 1).

Cellularised microspheres were incubated in Differentiation Medium 1, consisting of Dulbecco's Modified Eagle's Medium (DMEM) (Sigma-Aldrich, Dorset, UK), 2 \% horse serum (Gibco, Carlsbad, USA), 2 mM L-glutamine (Sigma-Aldrich, Dorset, UK), 100 U/ $\mathrm{mL}$ penicillin (Sigma-Aldrich, Dorset, UK), $100 \mu \mathrm{g} /$ $\mathrm{mL}$ streptomycin (Sigma-Aldrich, Dorset, UK) for $24 \mathrm{~h}$. After, Differentiation Medium 1 was replaced with $100 \mu \mathrm{L}$ Differentiation Medium 2 containing $15 \%$ horse serum in DMEM and changed every $2 \mathrm{~d}$ for $7 \mathrm{~d}$ (Pavlath, 1996) (Table 1).

\section{Measurement of HSMM cell proliferation}

HSMM cells were attached to TIPS microspheres in 24well ultra-low attachment plates under static-dynamic culture conditions. To each well $3 \mathrm{~mL}$ of proliferation medium was added and samples were incubated for 1 , 3,5 and $7 \mathrm{~d}$ with the culture medium changed every

Table 1. Composition of primary human skeletal muscle myoblast culture medium for proliferation and

\begin{tabular}{|l|l|l|}
\hline Proliferation medium & Differentiation medium 1 & Differentiation 2 \\
\hline Ham's F-10 Medium & Dulbecco's Modified Eagle Medium & Dulbecco's Modified Eagle Medium \\
$20 \%$ Foetal calf serum & $2 \%$ Horse serum & $15 \%$ Horse serum \\
$1 \%$ pen./strep. & $2 \mathrm{mM}$ L-glutamine & $2 \mathrm{mM} \mathrm{L-glutamine}$ \\
$10 \mathrm{ng} / \mathrm{mL} \mathrm{bFGF}$ & $100 \mathrm{U} / \mathrm{mL}$ penicillin & $100 \mathrm{U} / \mathrm{mL}$ penicillin \\
$0.1 \mu \mathrm{mol} / \mathrm{L}$ dexamethasone & $100 \mu \mathrm{g} / \mathrm{mL}$ streptomycin. & $100 \mu \mathrm{g} / \mathrm{mL}$ streptomycin. \\
\hline
\end{tabular}


2 d. A BrdU-ELISA assay was performed following the manufacturer's instructions (Roche, Basel, Switzerland). The microspheres were transferred from the ultra-low attachment plates into sterile microfuge tubes. To limit the interference of the microspheres, the amount of BrdUincorporated into proliferating cells in the HSMM attached to the TIPS microspheres was analysed using colourimetric detection of the supernatant product at $490 \mathrm{~nm}$ using a colourimetric plate reader (Multiskan, Thermo Scientific, Massachusetts, USA).

\section{In-cell Western analysis for skeletal muscle cell phenotype markers in HSMM attached to TIPS microspheres}

HSMM attached to the surfaces of TIPS microspheres incubated for either $1 \mathrm{~d}$ in proliferation medium, $7 \mathrm{~d}$ in proliferation medium, or $7 \mathrm{~d}$ in differentiation medium, or HSMM attached to tissue culture plastic for either $1 \mathrm{~d}$ in proliferation medium, $7 \mathrm{~d}$ in proliferation medium, or $7 \mathrm{~d}$ in differentiation medium, were washed in PBS followed by fixation in $4 \%$ formaldehyde in PBS for $30 \mathrm{~min}$. Cells were permeabilised by washing five times in $0.1 \%$ Triton X-100 in PBS for 5 min per wash. Non-specific binding was blocked using Odyssey blocking buffer (LiCor Biosciences, USA) for $1 \mathrm{~h}$ at room temperature. The samples were incubated with antibodies to Ki67 (0.2 mg/ $\mathrm{mL})$, Pax7 ( $1 \mathrm{mg} / \mathrm{mL})$, MyoD (1 mg/mL), myogenin (0.1 ng/mL) (Sigma-Aldrich, Dorset, UK), or skeletal muscle myosin heavy chain $(30 \mu \mathrm{g} / \mathrm{mL}$; Developmental Studies Hybridoma Bank, University of Iowa, Iowa, USA) at $4{ }^{\circ} \mathrm{C}$ overnight. Samples were washed extensively in PBS containing $0.1 \%$ Tween 20 five times for 5 min per wash. Samples were incubated with IR Dye $800 \mathrm{CW}$ secondary antibody (1:800) with CellTagTM 700 stain (1:500) (Li-Cor Biosciences, Cambridge, UK) in Odyssey blocking buffer for $1 \mathrm{~h}$ at room temperature. The TIPS microspheres did not cause interference with the infrared detection of the protein detection. The number of cells attached to the TIPS microspheres and in a monolayer was normalised using the CellTag'TM 700 stain, which was also used for well-to-well variation between the samples. Samples were extensively washed in PBS containing Tween 20 for 5 min per wash.
After a final wash all liquid was removed and the plate was scanned on the Odyssey Classic Infrared Imaging System (Li-Cor Biosciences, Cambridge, UK) using both $700 \mathrm{~nm}$ and $800 \mathrm{~nm}$ detection channels at $169 \mathrm{~nm}$ resolution, medium quality with a focus offset of $4.0 \mathrm{~mm}$. Quantitative in-cell Western analysis was performed using Image Studio Lite version 3.1 (Li-Cor Biosciences, Cambridge, UK).

\section{qPCR analysis for skeletal muscle cell phenotype markers in HSMM attached to TIPS microspheres}

TRIzol (Invtrogen, Paisley, UK) was used to extract RNA from HSMM attached to TIPS microspheres and incubated for either $1 \mathrm{~d}$ in proliferation medium, $7 \mathrm{~d}$ in proliferation medium, or $7 \mathrm{~d}$ in differentiation medium, or HSMM attached to tissue culture plastic either for $1 \mathrm{~d}$ in proliferation medium, $7 \mathrm{~d}$ in proliferation medium, or $7 \mathrm{~d}$ in differentiation medium. RNA content was quantified using a Nandrop 2000 system (Thermo Scientific, Surrey, UK). Total RNA was reverse transcribed using the Verso ${ }^{\text {TM }}$ cDNA kit (Thermo Scientific, Surrey, UK) to generate complementary DNA from total RNA. Samples were measured for $1 \mathrm{ng} / \mu \mathrm{L}$ total RNA. Real time PCR measurements were analysed relative to the internal control, GAPDH on a Roche LightCycler ${ }^{\circledR}$ II 480 system (Roche, Basel, Switzerland). Each reaction was performed in duplicate and contained $10 \mathrm{pmol}$ of specific primers (Table 2), SYBR Green Mastermix (Roche, Basel, Switzerland) and $1 \mu \mathrm{L}$ cDNA in a $20 \mu \mathrm{L}$ total reaction volume. A control consisting of water without cDNA was also included. Cycle threshold $(\mathrm{Ct})$ values were obtained for each gene of interest and calculated relative to GAPDH as the internal standard to be represented as $\Delta \mathrm{Ct}$ values. The calculated relative gene expression was normalised to controls of GAPDH expression level of 1.0.

\section{Aspect ratio analysis of HSMM on TIPS microspheres}

HSMM cells were attached to TIPS microspheres using static-dynamic incubation in 96-well plates. An equivalent number of cells per unit surface area were attached to the surface of $22 \mathrm{~mm}$ diameter gelatin-coated glass coverslips (VWR, Leicestershire, UK) (Table 3). Cells were incubated

Table 2. Human skeletal muscle myoblast forward and reverse primers for qPCR.

\begin{tabular}{|l|l|l|}
\hline Gene & Forward Primer & Reverse Primer \\
\hline Ki67 & AATACGTGAACAGGAGCCAGC & GTTGTTCCCTGAGCAACACTG \\
\hline Pax7 & ACTGTGCCCTCAGGTTTAGTG & CGTCGTCCTCCTTCTTGTCC \\
\hline MyoD & CTTGAGGGGCTAGGTTCACG & TGGGTTACGGTTACACCTGC \\
\hline Myogenin & GATCATCTGCTCACGGCTGA & CAGATGATCCCCTGGGTTG \\
\hline SkMHC & AGCGTGAGCTGGAAGATGTC & GCCCTCCTCTAGTCGGAAGA \\
\hline
\end{tabular}

Table 3. Calculation of cell seeding for accurate representation of cell number on the surface area of TIPS microsphere and tissue culture plastic control. The average number of cells attached per microsphere, within the 250-425 $\mu \mathrm{m}$ size range, was used to calculate the relative surface area coverage for a 12-well cover slip.

\begin{tabular}{|l|c|c|c|c|}
\hline Attachment object & Smallest microsphere & Largest microsphere & Average microsphere & 12-well coverslip \\
\hline Diameter $(\boldsymbol{\mu m})$ & 250 & 425 & 337.5 & 22,100 \\
\hline Radius $(\boldsymbol{\mu m})$ & 125 & 212.5 & 168.75 & 11050 \\
\hline Surface area $(\boldsymbol{\mu m})$ & 196,000 & 567,000 & 358,000 & $1,530,000,000$ \\
\hline Cell No. at $\mathbf{1} \mathrm{d}$ & & & 647 & $2,765,111$ \\
\hline
\end{tabular}


on TIPS microspheres for $1,3,5$ and $7 \mathrm{~d}$ under staticdynamic incubation conditions in proliferation medium. Culture medium was replaced every $2 \mathrm{~d}$. Cells were washed and fixed in $4 \%$ formaldehyde prior to phalloidin staining. Briefly, cells were washed, permeabilised in $0.1 \%$ for $5 \mathrm{~min}$ in Triton X-100, blocked in $1 \%$ bovine serum albumin for $30 \mathrm{~min}$ and incubated in Alexafluor-488 Phalloidin methanolic solution (Molecular Probes, Life Technologies, Carlsbad, USA) diluted in PBS for $20 \mathrm{~min}$ at room temperature. Samples were mounted onto a microscope slides in DAPI mountant (Vectashield, Vector Laboratories, Peterborough, UK). Images were acquired using a Leica SP8 confocal microscope (Leica, Solms, Germany) and the aspect ratio was calculated from cell length divided by cell width using the measurement tool in ImageJ.

\section{Statistical analysis}

All experiments were analysed in replicates of five of the same cells, unless stated otherwise. The data were analysed using the unpaired two-tail Student's $t$-test and one-way ANOVA using GraphPad Prism v.6.00 statistical software (GraphPad Software, San Diego, USA). The data were presented as mean \pm standard deviation. A $p$ value of $<0.05$ indicated statistical significance.

\section{Results}

\section{Proliferation of HSMM Attached to TIPS microspheres}

The proliferation of HSMM cultured on TIPS microspheres in proliferation medium at days 1, 3,5 and 7 was measured using a BrdU-ELISA. The assay was performed on aliquots of TIPS microspheres incubated under static-dynamic conditions (Fig. 1). The proportion of proliferating HSMM cells remained consistent when cultured on TIPS microspheres over $7 \mathrm{~d}$. No significant difference was observed between groups.

\section{Characterisation of phenotypic status of HSMM on TIPS microspheres}

In-cell Western analysis of skeletal muscle cell phenotype markers

HSMM cells were cultured on TIPS microspheres for 1 and $7 \mathrm{~d}$ in proliferation medium and $7 \mathrm{~d}$ in differentiation medium. Cells incubated on conventional flat tissue culture substrates for each group were analysed in parallel. Protein expression of myogenic regulatory factors in HSMM under proliferation and differentiation conditions was measured using in-cell Western analysis and quantified relative to cell number using the housekeeping CellTag $700^{\mathrm{TM}}$ protein. The signal intensity for each specific protein probed for was calculated per cell by normalising signal to cell number (Fig. 2a-e). Myoregulatory factor protein expression in HSMM attached to TIPS microspheres incubated in differentiation medium for $7 \mathrm{~d}$ exhibited higher values for Ki67 $(p<0.01), \operatorname{Pax} 7(p<0.005), \operatorname{MyoD}(p<0.001)$, myogenin $(p<0.001)$ and skeletal muscle myosin heavy chain $(p<0.001)$ compared with HSMM attached to TIPS microspheres at day 1. HSMM attached to TIPS

\section{HSMM Proliferation on TIPS Microspheres}

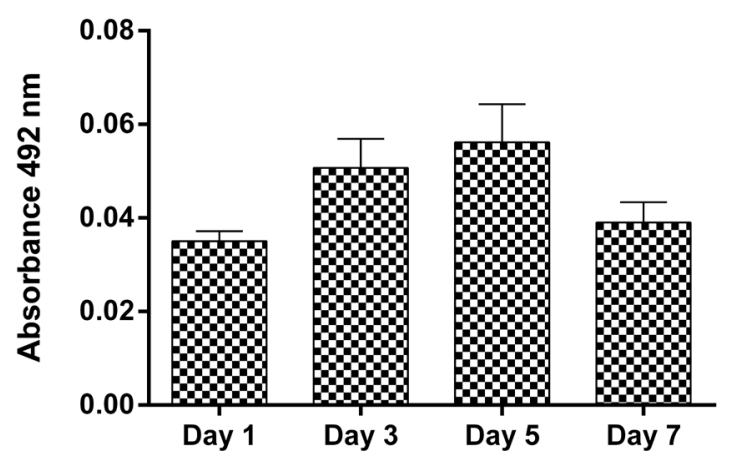

Fig. 1. Proliferation of human skeletal muscle myoblasts on TIPS microspheres over 1-7 d in proliferation medium under static-dynamic incubation conditions. TIPSattached myoblasts showed consistent proliferation over $7 \mathrm{~d}$ in proliferation medium.

microspheres and incubated in proliferation medium for 1 or $7 \mathrm{~d}$ showed no difference in myoregulatory factor expression between the different time points.

qPCR analysis of skeletal muscle cell phenotype markers The phenotypic status of HSMM attached to TIPS microspheres was quantitatively analysed for myoregulatory factor expression using qPCR. HSMM attached to TIPS microspheres were incubated in proliferation medium for 1 or $7 \mathrm{~d}$, and in differentiation medium for $7 \mathrm{~d}$ before qPCR was performed using relative quantification at day 1 . Control groups consisting of cells attached to conventional tissue culture plastic surfaces were also analysed. All data were compared to GAPDH gene expression and compared with the Ct cycle of the calibrator obtained with the cells incubated on TIPS microspheres for $1 \mathrm{~d}$ (Fig. 3a-e). The expression of Ki67 did not change in HSMM cells attached to TIPS microspheres between days 1-7. HSMM attached to TIPS microspheres expressed Pax 7, MyoD and myogenin at significantly higher levels in samples incubated for $7 \mathrm{~d}$ in proliferation and differentiation medium compared with the expression at day 1 . The relative expression of skeletal muscle myosin heavy chain in HSMM attached to TIPS microspheres cultured for $7 \mathrm{~d}$ in differentiation medium resulted in a 14-fold increase compared with expression in HSMM on TIPS microspheres at day 1.

\section{Aspect ratio analysis of HSMM on TIPS microspheres}

The effect of culturing HSMM on TIPS microspheres on the cell morphology was explored using cell aspect ratio analysis of phalloidin-stained cells and compared with HSMM attached to tissue culture plastic surfaces (Fig. 4). Quantitative analysis of aspect ratio indicated that cells incubated in proliferation medium and differentiation medium for $7 \mathrm{~d}$ had a higher aspect ratio $(p<0.01)$, compared with cells cultured on tissue culture plastic under the same conditions. Confocal microscopy revealed the cell cytoskeleton to be morphologically elongated, when cultured on TIPS microspheres for $7 \mathrm{~d}$, compared with 


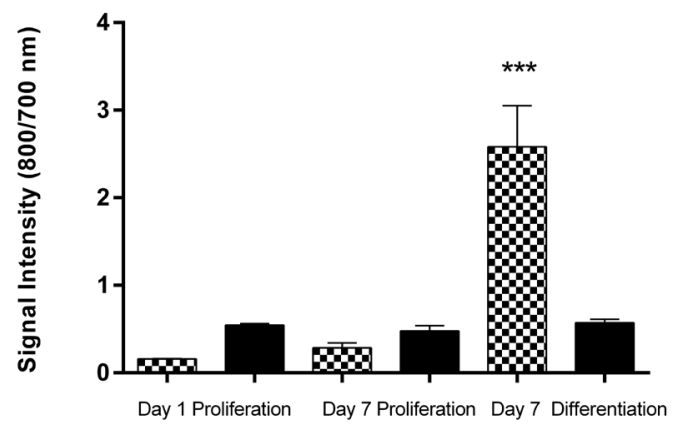

\% HSMM-TIPS Microspheres

HSMM-Tissue Culture Plastic

Day 1 Proliferation Day 7 Proliferation Day 7 Differentiation

Pax7 Expression in HSMM on TIPS Microsphere vs. TC Plastic

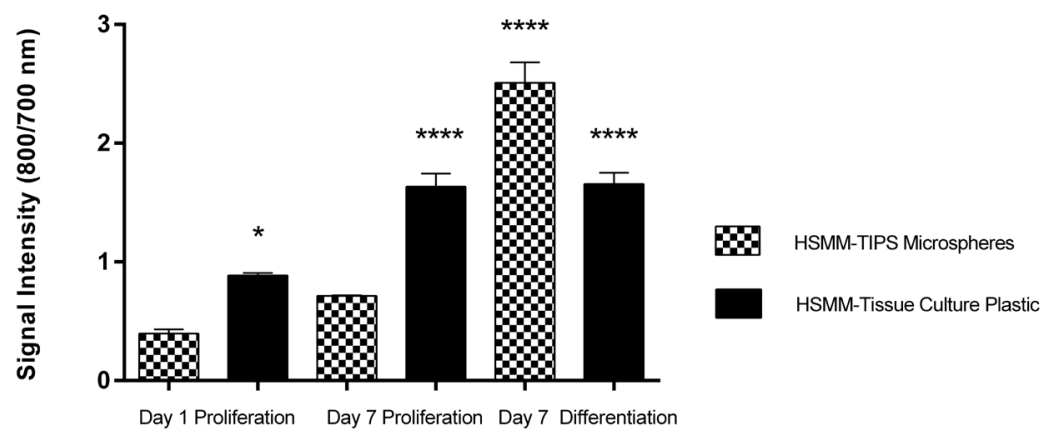

MyoD Expression in HSMM on TIPS Microsphere vs. TC Plastic

c

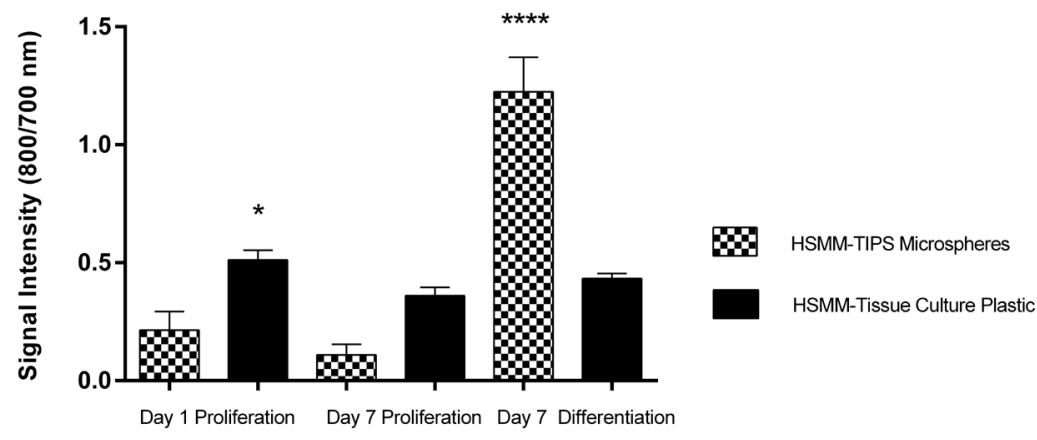

Myogenin Expression in HSMM on TIPS Microsphere vs. TC Plastic

d

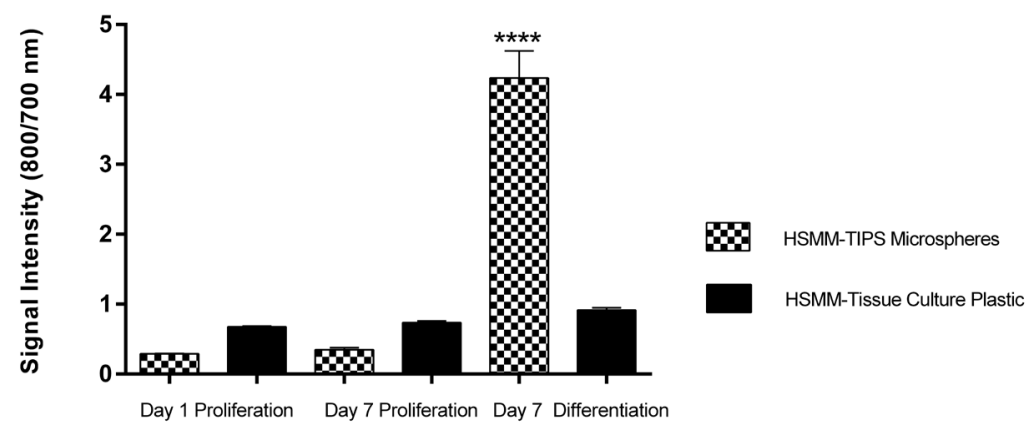

Fig. 2. Protein expression levels of (a) Ki67, (b) pax7, (c) MyoD, (d) myogenin and (e) skeletal muscle myosin heavy chain in HSMM attached to TIPS microspheres versus expression in HSMM attached to tissue culture plastic surfaces Relative expression in all groups was compared with HSMM attached to TIPS microspheres after $1 \mathrm{~d}$ in proliferation medium $(* p<0.05, * * p<0.01, * * * p<0.005$, $* * * * p<0.001)$.

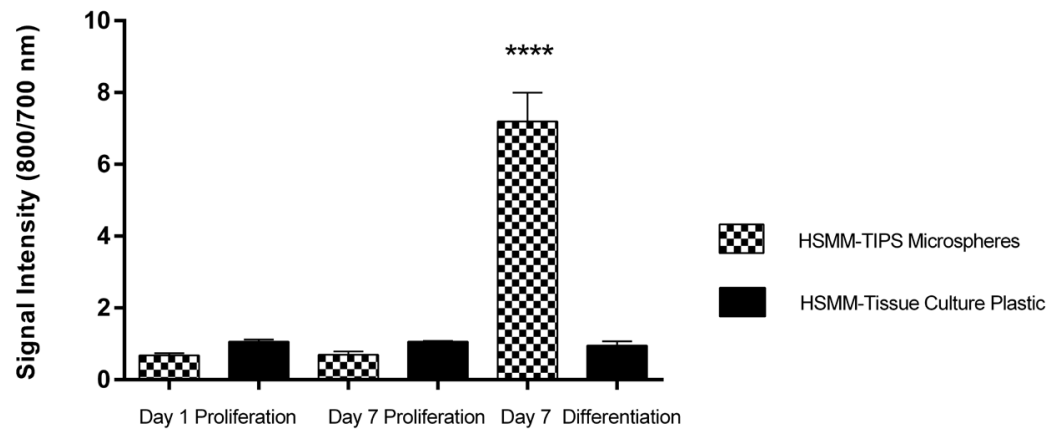


Ki67 Expression in HSMM on TIPS Microspheres vs.

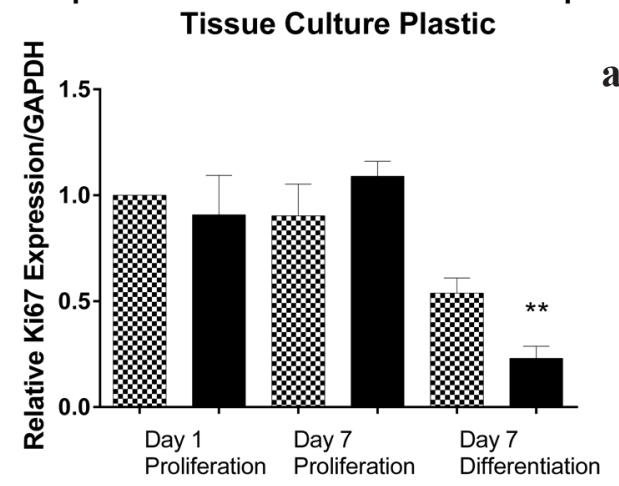

W HSMM-TIPS Microspheres HSMM on Tissue Culture Plastic

MyoD Expression in HSMM on TIPS Microspheres vs.

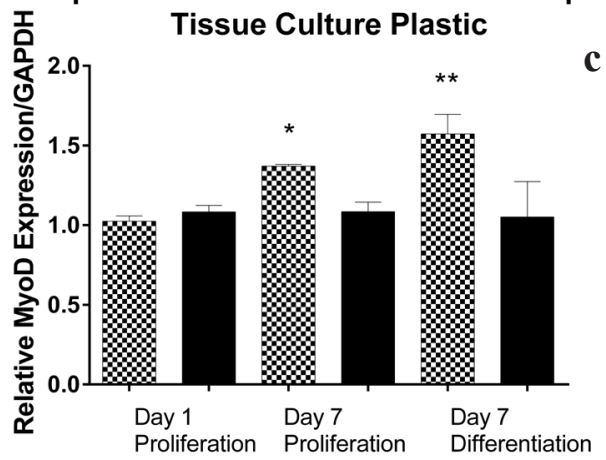

3. HSMM-TIPS Microspheres HSMM on Tissue Culture Plastic

Fig. 3. mRNA expression levels of (a) Ki67, (b) pax7, (c) MyoD, (d) myogenin and (e) skeletal muscle myosin heavy chain mRNA in HSMM attached to TIPS microspheres versus expression in HSMM attached to tissue culture plastic surfaces. HSMM cultured on tissue culture plastic in proliferation medium for 1 and $7 \mathrm{~d}$ or in differentiation medium for $7 \mathrm{~d}$ served as controls. Relative expression in all groups was compared with HSMM attached to TIPS microspheres after $1 \mathrm{~d}$ in proliferation medium $(* p<0.05$, $* * p<0.01, * * * p<0.005, * * * * p<0.001)$.

\section{Pax7 Expression in HSMM on TIPS Microspheres vs.}

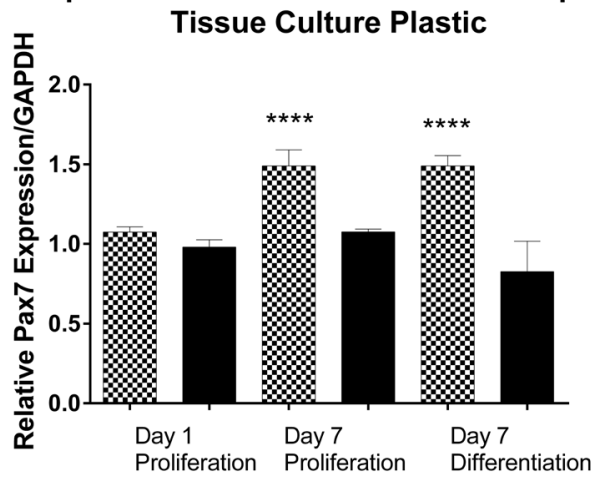

\$ HSMM-TIPS Microspheres

HSMM on Tissue Culture Plastic
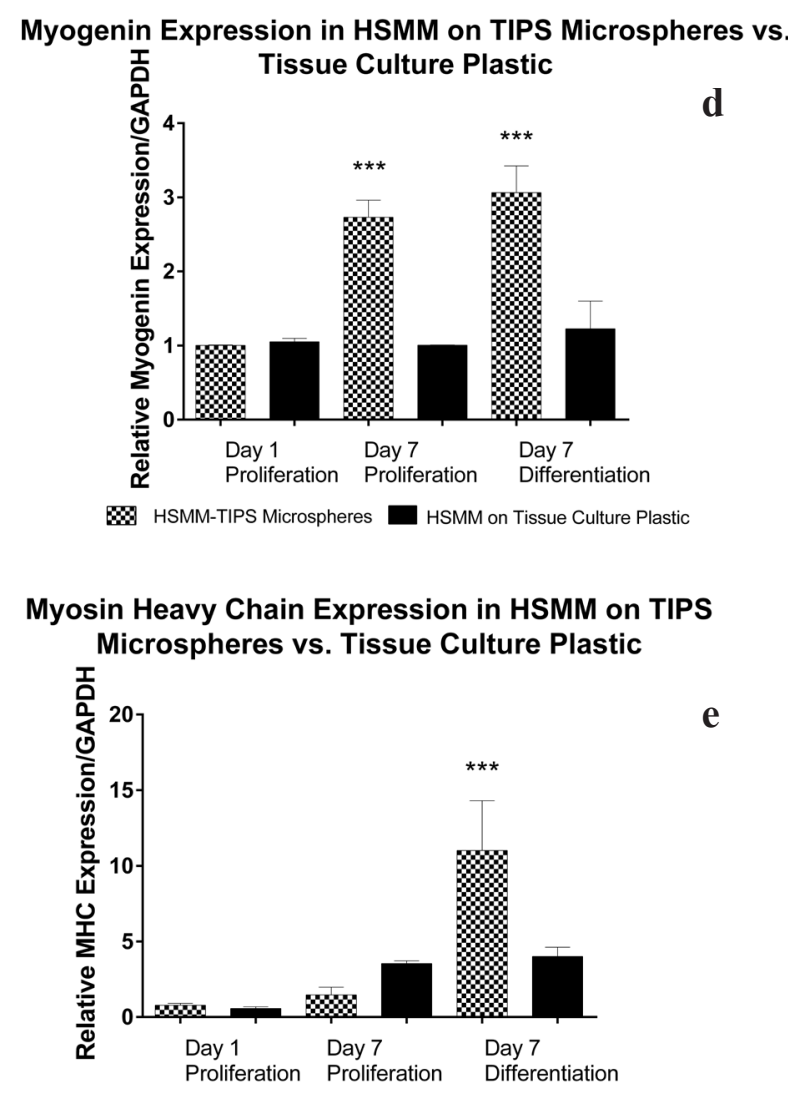

\& HSMM-TIPS Microspheres the cytoskeleton arrangement of cells cultured on tissue culture plastic for the same period of time. Cells cultured on tissue culture plastic in differentiation medium for $7 \mathrm{~d}$ were also significantly elongated, compared with cells cultured on tissue culture plastic for $1 \mathrm{~d}$ in differentiation or proliferation medium $(p<0.05)$.

\section{Discussion}

\section{Characterisation of HSMM attached to TIPS microspheres}

Delivery of myoblasts to treat skeletal muscle injury often requires cell expansion in vitro. Cell delivery using 3D scaffolds has previously been used for proliferation in vitro (Knight et al., 2013; Chang et al., 2007). The role of a scaffold is to provide a template for regenerating tissue and may influence cell behaviour. This includes cell attachment, migration, proliferation, and differentiation to a specific cell phenotype, which could impact on the rate of tissue formation and efficacy of the treatment (Smith et al., 2009; Wei and Ma, 2008). In the current study the effect of attaching HSMM cells to TIPS microspheres was investigated in the context of their phenotypic status. A proliferation assay, measuring incorporation of BrdU into DNA of proliferating cells, revealed no significant change to cell proliferation during 1-7 d incubation in proliferation medium. This finding would suggest that HSMM retain their proliferative status when cultured on the surface of TIPS microspheres and do not spontaneously differentiate. This could be of value during a bioprocessing and therapeutic setting, which may facilitate tissue regeneration 
a
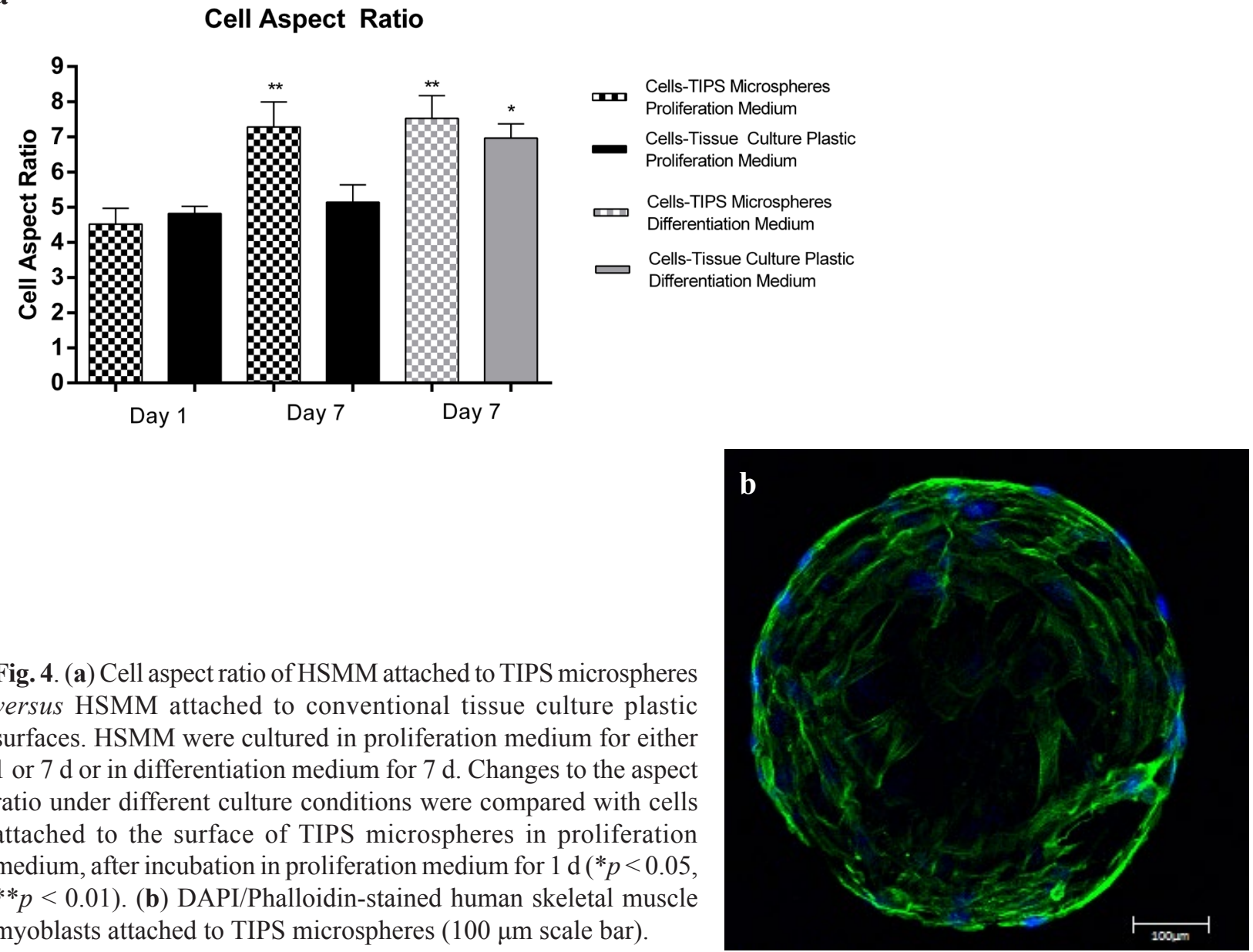

Fig. 4. (a) Cell aspect ratio of HSMM attached to TIPS microspheres versus HSMM attached to conventional tissue culture plastic surfaces. HSMM were cultured in proliferation medium for either 1 or $7 \mathrm{~d}$ or in differentiation medium for $7 \mathrm{~d}$. Changes to the aspect ratio under different culture conditions were compared with cells attached to the surface of TIPS microspheres in proliferation medium, after incubation in proliferation medium for $1 \mathrm{~d}\left({ }^{*} p<0.05\right.$, $* * p<0.01$ ). (b) DAPI/Phalloidin-stained human skeletal muscle myoblasts attached to TIPS microspheres (100 $\mu \mathrm{m}$ scale bar).

in a clinical setting. A void of defective tissue requires a sufficient mass of cells for replacement of the previously lost injured tissue.

\section{Manipulation of phenotypic lineage}

A current challenge for cell based therapy products is the control of cell fate upon transplantation (Ankrum et al., 2014). For muscle regeneration, it is currently uncertain whether it is preferable for cells to be transplanted in a proliferative state as myoblasts, or differentiated as myotubes or myofibres. In culture, the presence of myotubes will eventually induce the cessation of cell proliferation. In vivo, this could also be influenced by the release of cytokines and other factors present at the site of injury (Chiron et al., 2012). The current study demonstrates it is possible to manipulate the phenotype of myoblasts attached to TIPS microspheres in vitro under defined conditions.

Myoblast differentiation was initiated by the addition of $2 \%$ horse serum, which leads to an increased level of MyoD that stimulates creatine phosphokinase activity and formation of contractile apparatus (Zalin, 1972; Lawson and Purslow, 2000; Yeh et al., 2014). Cells attached to TIPS microspheres, cultured in differentiation medium, exhibited an increase in myoregulatory factors Ki67, pax7, myogenin, and myosin heavy chain. The co-expression of markers of proliferation and differentiation after incubation in differentiation medium, suggests that these incubation conditions result in phenotypic heterogeneity in the cells attached to the microspheres. The mixed phenotype corresponds with data available from the cell product manufacturer that indicated the cells were $>60 \%$ desmin positive (Lonza). Delivery of a phenotypically heterogeneous population of HSMM on TIPS microspheres may be beneficial, by offering engraftment of cells with contractile apparatus as well as more proliferative cells that could further increase the number of cells at the target site. Interestingly, MyoD expression was not increased in HSMM attached to TIPS microspheres incubated in differentiation medium. Previous studies have shown that in a heterogeneous population of myogenic cells, MyoD expression may be reduced as reserve cells are generated to delay further differentiation (Yoshida et al., 1998).

Myoblast differentiation is a multifaceted process that implicates withdrawal from the cell cycle and morphological changes that include elongation, alignment and fusion of myoblasts to form multinucleated myofibres. HSMM mRNA analysis of myogenic regulatory factors demonstrated increased expression of myogenin and myosin heavy chain in cells cultured on tissue culture plastic in differentiation medium. This was translated to a significant increase in the protein expression of myosin heavy chain in HSMM attached to TIPS incubated in differentiation medium for $7 \mathrm{~d}$. The increased expression 
of myosin heavy chain in HSMM cells attached to the microspheres may be due to differences in the surface topography or substrate stiffness offered by microspheres compared with tissue culture plastic. Substrate stiffness has important implications for muscle regeneration strategies. For example, sarcomeres will not form in vitro unless myoblasts grow on a surface with stiffness representative of physiological muscle stiffness (Engler et al., 2004). The experiments conducted were based on repeats from one cell donor. Moving forward with this research will require further characterisation of cells attached to the TIPS microspheres using different cell donors.

Delivery of myoblasts on a 3D structure may enhance the survival of transplanted cells. A current limitation for many cell based therapies is the lack of a sufficient vascular supply to sites of cell transplantation (De Coppi et al., 2005). It is estimated that cells need to be no more than $0.1 \mathrm{~mm}$ away from a capillary to receive oxygen and nutrients as well as to remove waste products (Goodsell, 2003). Packing of the TIPS microspheres results in an open scaffold structure with predictable interstices between adjacent particles. This will facilitate the passage of nutrients and waste, increasing the likelihood of cell survival.

\section{Cell elongation on TIPS microspheres}

The current study investigated changes to the aspect ratio of myoblasts attached to TIPS microspheres under proliferation and differentiation conditions. Cell aspect ratio is calculated by dividing cell height by cell width and used to quantify cytoskeletal arrangement. This has previously been used by (Crouch et al., 2009), to study the anisotropic and geometric cell behaviour in relation to biomaterial surface topography (Crouch et al., 2009). HSMM were observed to have elongated on TIPS microspheres after $7 \mathrm{~d}$ culture in proliferation and differentiation medium. There was no difference in the elongation of cells in proliferation medium at day 1 on TIPS microspheres compared with cells on tissue culture plastic at days 1 and 7 in proliferation medium. Therefore, the cytoskeletal changes observed may have resulted from the initiation of differentiation cues. Elongation of cells in proliferation medium at day 7 indicates that some cells undergo spontaneous differentiation on TIPS microspheres. This may account for apparent differences of myoblasts incubated on TIPS, compared with on monolayer surfaces. The cause of this is unknown, but the $3 \mathrm{D}$ topography of the microsphere surface may elicit similar cues to the ECM. Cellular morphology is directed by the ECM and is thought to be an inherent cue to control myogenic differentiation, regardless of the use of chemical induction factors (Chiron et al., 2012; Baker, 2012).

\section{Cell delivery via TIPS microspheres}

Delivery of cell suspensions derived from conventional methods using 2D tissue culture substrates, requires their detachment from the culture vessel prior to delivery. This process interferes with cell adhesion to extracellular matrix proteins and may disrupt cell membranes, resulting in changes to cell viability and function (Gilbert et al., 2006; Yang et al., 2007; Ahmadi et al., 2011). Such detrimental effects could diminish the efficacy of the transplanted cell product and contribute to poor clinical outcomes. In this study, TIPS microspheres were tested as a substrate for the attachment of anchorage-dependent cells. The migration of cells from the site of implantation into other tissues may be reduced by the targeted delivery of cells using TIPS microspheres, resulting in potentially improved efficiency of cell number. TIPS microspheres possess a large surface pore, providing an internal cavity and increased surface area. The internal cavity feature promotes host tissue infiltration into the microsphere, to avoid it being extruded from the implantation site (Blaker et al., 2008a; Keshaw et al., 2010). Thus, the migration of transplanted cells from the site of injury is likely to be reduced. Improved host-tissue infiltration into the microsphere cavity, along with diffusion of oxygen and nutrients to the transplanted cells is likely to facilitate engraftment and cell survival.

\section{Future directions}

Effective regeneration of volumetric muscle loss, using the type of approach outlined in the current study, will require further understanding of the optimal phenotypic distribution of cells being delivered so that they match the conditions of the target site. This is likely to differ, depending on the condition being treated. Further studies will also be necessary, to determine whether the transplanted cells differentiate and provide functional contractile properties similar to native skeletal muscle.

\section{Conclusion}

Results from the current study demonstrate that the phenotypic status of myoblasts, attached to TIPS microspheres, can be controlled independently to the delivery of conventional chemical stimuli. The efficient attachment of myoblasts to the microspheres and their continued proliferation offers an assistive technology for minimally invasive delivery of cells for muscle therapy.

\section{Acknowledgements}

The project was supported by generous grants from The Sir Halley Stewart Trust, The Henry Smith Charity, and the UCL Grand Challenge Studentship Scheme. The authors appreciate the technical support of Veronika Kallo and Professor Surjit Kaila Srai, UCL Structural and Molecular Biology. This work was undertaken at UCL/UCLH which receives funding from the Department of Health's NIHR as a Comprehensive Biomedical Research Centre.

\section{References}

Ahmadi R, Mordan N, Forbes A, Day RM (2011) Enhanced attachment, growth and migration of smooth muscle cells on microcarriers produced using thermally induced phase separation. Acta Biomater 7: 1542-1549.

Ankrum JA, Miranda OR, Ng KS, Sarkar D, Xu C, Karp JM (2014) Engineering cells with intracellular agent- 
loaded microparticles to control cell phenotype. Nat Protoc 9: 233-245.

Baker BM, Chen CS (2012) Deconstructing the third dimension: how 3D culture microenvironments alter cellular cues. J Cell Sci 125: 3015-3024.

Blaker JJ, Knowles JC, Day RM (2008a) Novel fabrication techniques to produce microspheres by thermally induced phase separation for tissue engineering and drug delivery. Acta Biomater 4: 264-272.

Blaker JJ, Pratten J, Ready D, Knowles JC, Forbes A, Day RM (2008b) Assessment of antimicrobial microspheres as a prospective novel treatment targeted towards the repair of perianal fistulae. Aliment Pharmacol Ther 28: 614-622.

Chang W, Lim S, Song H, Lee S, Song BW, Jang Y, Chung N, Hwang KC (2007) In vitro Expansion of mesenchymal stem cells using 3-D matrix derived from cardiac fibroblast. Tissue Engineering and Regenerative Medicine 4: 370-375.

Chiron S, Tomczak C, Duperray A, Laine J, Bonne G, Eder A, Hansen A, Eschenhagen T, Verdier C, Coirault C (2012) Complex interactions between human myoblasts and the surrounding 3D fibrin-based matrix. PLoS One 7: e36173. doi: 10.1371 /journal.pone.0036173.

Costa ML (2014) Cytoskeleton and adhesion in myogenesis. ISRN Developmental Biology 2014: Article ID 713631, 15 pages, 2014. doi:10.1155/2014/713631.

Crouch AS, Miller D, Luebke KJ, Hu W (2009) Correlation of anisotropic cell behaviors with topographic aspect ratio. Biomaterials 30: 1560-1567.

De Coppi P, Delo D, Farrugia L, Udompanyanan K, Yoo JJ, Nomi M, Atala A, Soker S (2005) Angiogenic genemodified muscle cells for enhancement of tissue formation. Tissue Eng 11: 1034-1044.

Engler AJ, Griffin MA, Sen S, Bonnemann CG, Sweeney HL, Discher DE (2004) Myotubes differentiate optimally on substrates with tissue-like stiffness: pathological implications for soft or stiff microenvironments. J Cell Biol 166: 877-887.

Gilbert TW, Sellaro TL, Badylak SF (2006) Decellularization of tissues and organs. Biomaterials 27: 3675-3683.

Goodsell DS (2003) The molecular perspective: VEGF and angiogenesis. Stem Cells 21: 118-119.

Kang SW, Seo SW, Choi CY, Kim BS (2008) Porous poly(lactic-co-glycolic acid) microsphere as cell culture substrate and cell transplantation vehicle for adipose tissue engineering. Tissue Eng Part C Methods 14: 25-34.

Keshaw H, Thapar N, Burns AJ, Mordan N, Knowles JC, Forbes A, Day RM (2010) Microporous collagen spheres produced via thermally induced phase separation for tissue regeneration. Acta Biomater 6: 1158-1156.

Kilian KA, Bugarija B, Lahn BT, Mrksich M (2010) Geometric cues for directing the differentiation of mesenchymal stem cells. Proc Natl Acad Sci U S A 107: 4872-4877.

Knight T, Basu J, Rivera EA, Spencer T, Jain D, Payne R (2013) Fabrication of a multi-layer three-dimensional scaffold with controlled porous micro-architecture for application in small intestine tissue engineering. Cell Adh Migr 7: 267-274.
Lawson MA, Purslow PP (2000) Differentiation of myoblasts in serum-free media: effects of modified media are cell line-specific. Cells Tissues Organs 167: 130-137.

Pavlath GK (1996) Isolation, purification, and growth of human skeletal muscle cells. Methods Mol Med 2: $307-$ 317.

Przewozniak M, Czaplicka I, Czerwinska AM, Markowska-Zagrajek A, Moraczewski J, Streminska W, Janczyk-Ilach K, Ciemerych MA, Brzoska E (2013) Adhesion proteins - an impact on skeletal myoblast differentiation. PLoS One 8: e61760. doi: 10.1371/journal. pone.0061760.

Schwander M, Leu M, Stumm M, Dorchies OM, Ruegg UT, Schittny J, Muller U (2003) Betal integrins regulate myoblast fusion and sarcomere assembly. Dev Cell 4: 673-685.

Sekiya EJ, Forte A, Kuhn TI, Janz F, Bydlowski SP, Alves A (2012) Establishing a stem cell culture laboratory for clinical trials. Rev Bras Hematol Hemoter 34: 236-241.

Smith IO, Liu XH, Smith LA, Ma PX (2009) Nanostructured polymer scaffolds for tissue engineering and regenerative medicine. Wiley Interdiscip Rev Nanomed Nanobiotechnol 1: 226-236.

Wei G, Ma PX (2008) Nanostructured biomaterials for regeneration. Adv Funct Mater 18: 3566-3582.

Yang Y, Rossi FM, Putnins EE (2007) Ex vivo expansion of rat bone marrow mesenchymal stromal cells on microcarrier beads in spin culture. Biomaterials 28: 3110-3120.

Yao L, Phan F, Li Y (2013) Collagen microsphere serving as a cell carrier supports oligodendrocyte progenitor cell growth and differentiation for neurite myelination in vitro. Stem Cell Res Ther 4: 109.

Yeh TS, Hsu CC, Yang SC, Hsu MC, Liu JF (2014) Angelica Sinensis promotes myotube hypertrophy through the PI3K/Akt/mTOR pathway. BMC Complement Altern Med 14: 144.

Yoshida N, Yoshida S, Koishi K, Masuda K, Nabeshima Y (1998) Cell heterogeneity upon myogenic differentiation: down-regulation of MyoD and Myf-5 generates 'reserve cells'. J Cell Sci 111: 769-779.

Zalin R (1972) Creatine kinase activity in cultures of differentiating myoblasts. Biochem J 130: 79P.

\section{Discussion with Reviewers}

S. Richardson: The authors highlight that the TIPS microspheres have an internal cavity, which they suggest would promote host tissue infiltration. Can the authors speculate as to whether the cavity will influence local adhered cell phenotype compared to cells on the outer surface, possibly due to differences in cell density or local cell-cell interactions?

Authors: To date, this specific area has not been studied in terms of the phenotypic status of the attached cells. This unknown may cause induced differentiation of cells which are likely to be in closer proximity to each other. However, as the cells are likely to be seeded as a monolayer in the cavity the cells are likely to be exposed to similar local cell-cell interactions as on the outer layer. 
U. Rüegg: What will be done in order to investigate whether this technique will provide functional improvement of skeletal muscle?

Authors: The next stage of development for this approach is to demonstrate the functional restoration of injured skeletal muscle. The group is currently investigating strategies to deliver myoblasts attached to the surface of the microparticles to a discrete skeletal muscle injury.

R. Oldershaw: How do you envisage the application of the TIPS microparticles within the context of other clinical indications?

Authors: This composition of TIPS microparticles has been demonstrated to promote excellent cell attachment properties for a variety of anchorage-dependent cell types. For some conditions which require targeted cell delivery, the delivery of cells on the surface of TIPS microparticles may promote more localised repair and regeneration. Current cell therapy approaches to conditions such as type II diabetes, localised muscular dystrophies and myocardial infarction usually require the systemic delivery of a high number of cells in suspension to account for cell death and non-specific delivery. The unique advantages of the TIPS microparticles permit the targeted delivery of cells to a discrete injury, using a minimally invasive approach.

Editor's Note: Scientific Editor in charge of the paper: Jürg Gasser. 